\title{
Editorial
}

\section{International Conference on Advances in Mechanical Engineering and Mechanics 2010}

\author{
Sami El-Borgi ${ }^{1,2}$ \\ ${ }^{1}$ Mechanical Engineering Program, Texas A\&M University at Qatar, Doha, Qatar \\ ${ }^{2}$ Applied Mechanics and Systems Research Laboratory, Tunisia Polytechnic School, University of Carthage, La Marsa, Tunisia \\ Correspondence should be addressed to Sami El-Borgi; sami.el_borgi@qatar.tamu.edu
}

Received 3 October 2013; Accepted 3 October 2013; Published 8 May 2014

Copyright (C) 2014 Sami El-Borgi. This is an open access article distributed under the Creative Commons Attribution License, which permits unrestricted use, distribution, and reproduction in any medium, provided the original work is properly cited.

The present special issue of Shock and Vibration journal includes six papers presenting studies conducted in various fields of vibration. Papers were selected among those presented at the Fifth International Conference on Advances in Mechanical Engineering and Mechanics 2010 (ICAMEM 2010) held on December 18-20, 2010, in Hammamet, Tunisia. All papers were reviewed by distinguished reviewers according to the journal's procedures and standards. Among the vast number of papers that were presented at the conference, the following studies were selected for this special issue.

Samaali et al. study the response of a capacitive MEMS switch composed of two clamped-clamped flexible microbeams. An electrostatic force is applied between the microbeams to yield the switch to its ON and OFF states. The equations of motion of the system and the associated boundary conditions are derived and the static and dynamic problems are solved using the differential quadratic method. The obtained analytical results are validated using numerical finite element results. The transient behavior of the microswitch was then investigated, indicating a reduction in actuation voltage, switching time, and power consumption while maintaining a relatively good RF performance.

Jemai et al. develop a mathematical model that accurately simulates the dynamic behavior of active fiber composites (AFC). These composites are known for their flexibility and relatively high actuation capacity compared with traditional ceramic piezoelectric materials. The AFC's energy harvesting capabilities, however, are low. The model uses homogenization techniques to describe piezoelectric properties of the AFC. The developed model is incorporated into a vibrationbased energy harvesting system consisting on a cantilever beam on top of which an AFC patch is attached. Analytical solutions of the dynamic behavior and the harvested voltage are proposed and validated with finite element simulations.

Gafsi et al. propose a new passive control strategy for the vibration confinement in a flexible nonlinear beam by considering an inverse eigenvalue problem. The strategy consists of determining the geometric parameters of a beam to yield a desired set of mode shapes and natural frequencies. The beam dynamics is described by a nonlinear partial differential equation that is linearized. The authors show that, using the linear model, the strategy of vibration confinement remains valid for the nonlinear beam. They also show that having higher amplitude on a larger frequency interval in conjunction with significant level of vibration confinement on a smaller region of the spatial domain presents an efficient design for energy harvesting.

El Ouni and Ben Kahla investigate numerically the active tendon control of a cable-stayed bridge in a construction phase. A linear finite element model of a small-scale mock-up of the bridge is first developed, coupled with a geometrically nonlinear model for the cable. Active damping is added to the structure by using pairs of collocated force actuatordisplacement sensors located on each active cable and controlled by decentralized first order positive position feedback (PPF) or direct velocity feedback (DVF). A decentralized parallel PPF-DVF is proposed to obtain the best performance from the two compensators. The authors show that the proposed strategy can be used to control both deck and cable vibrations induced by parametric excitation.

Jiang et al. propose a methodology for fault diagnosis of rotating machinery based on vibration multisensor 
information fusion. Three case studies are used to show the effectiveness of the proposed methodology, namely, diagnostic of faulty gear, rolling bearing, and identification of rotor crack. For each case study, the sensibilities of the features are analyzed. The obtained results indicate that the peak factor is the most sensitive feature for identifying gear defect, among the twelve time-domain features that are considered. The mean, amplitude squared, root mean square, root amplitude, and standard deviation are all suitable for identifying gear, rolling bearing, and rotor crack defect on a comparative basis.

In identifying the modal signature of structures based on output-only measurements, several algorithms have been developed over the years among which is the family of stochastic subspace identification techniques known for their robustness and convergence. For large-scale structures, the selection of the model order and the corresponding system poles is often challenging. To address this problem, the concept of the "stabilization diagram" is introduced. The problem with these diagrams is that the resulting spurious modes need to be eliminated. Mrabet et al. suggest a new stabilization criterion obtained through a novel numerical implementation of the stabilization diagram. The new implementation makes the alignment of the stabilization diagram more robust, so that only the spurious modes are removed. The authors apply the new stabilization diagram and the implemented identification technique to identify the modal signature of an aircraft structure. 

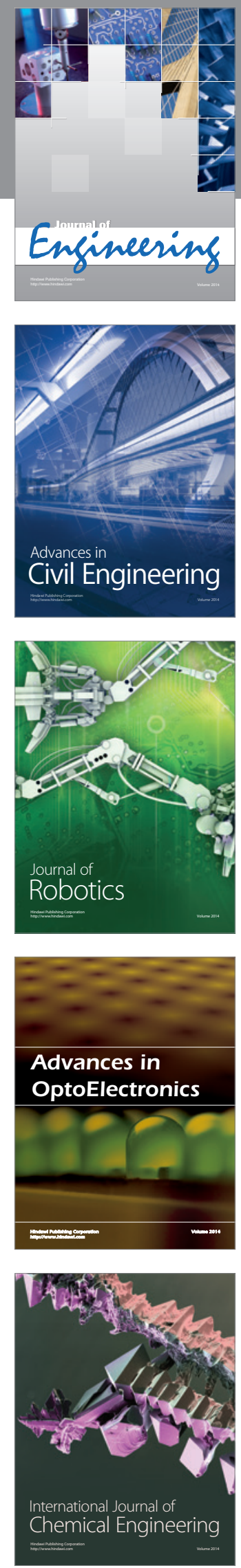

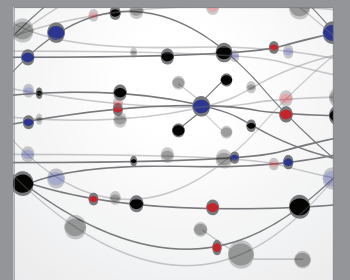

The Scientific World Journal
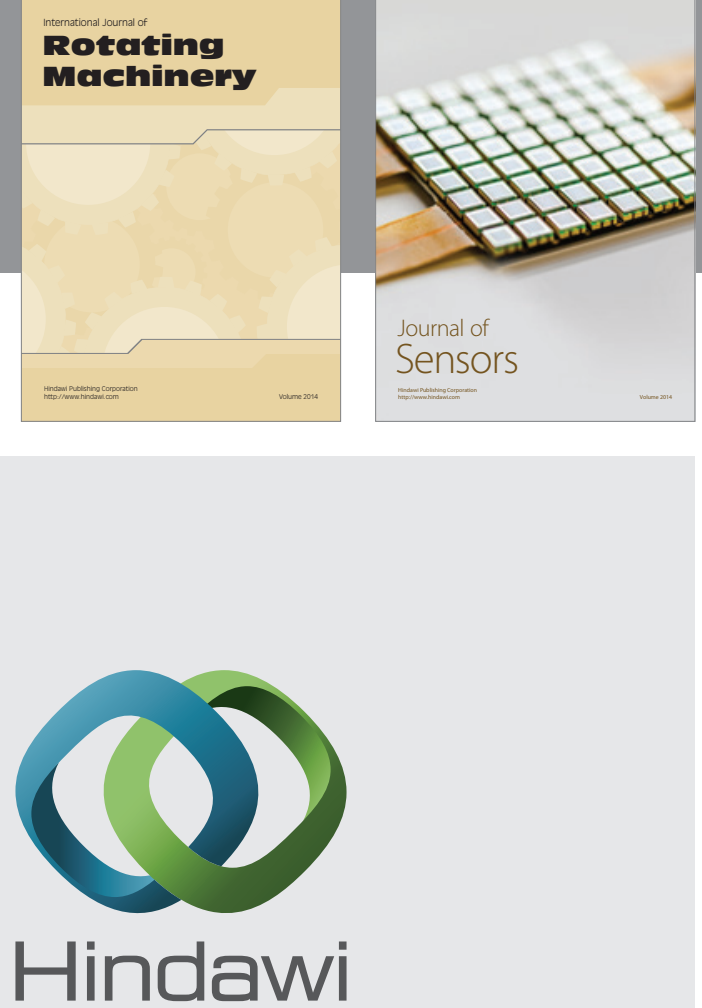

Submit your manuscripts at http://www.hindawi.com
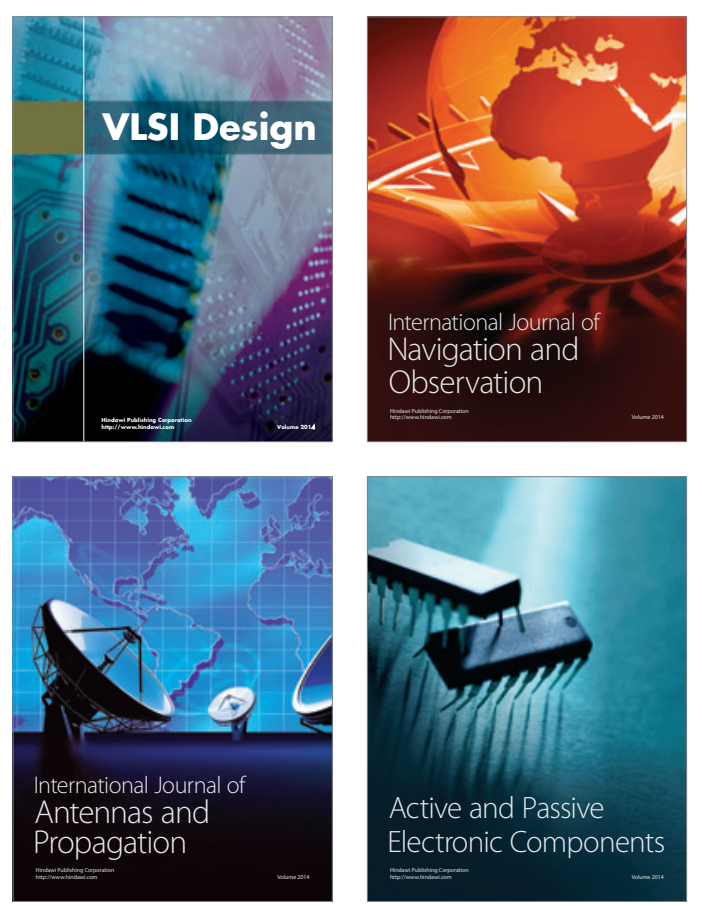
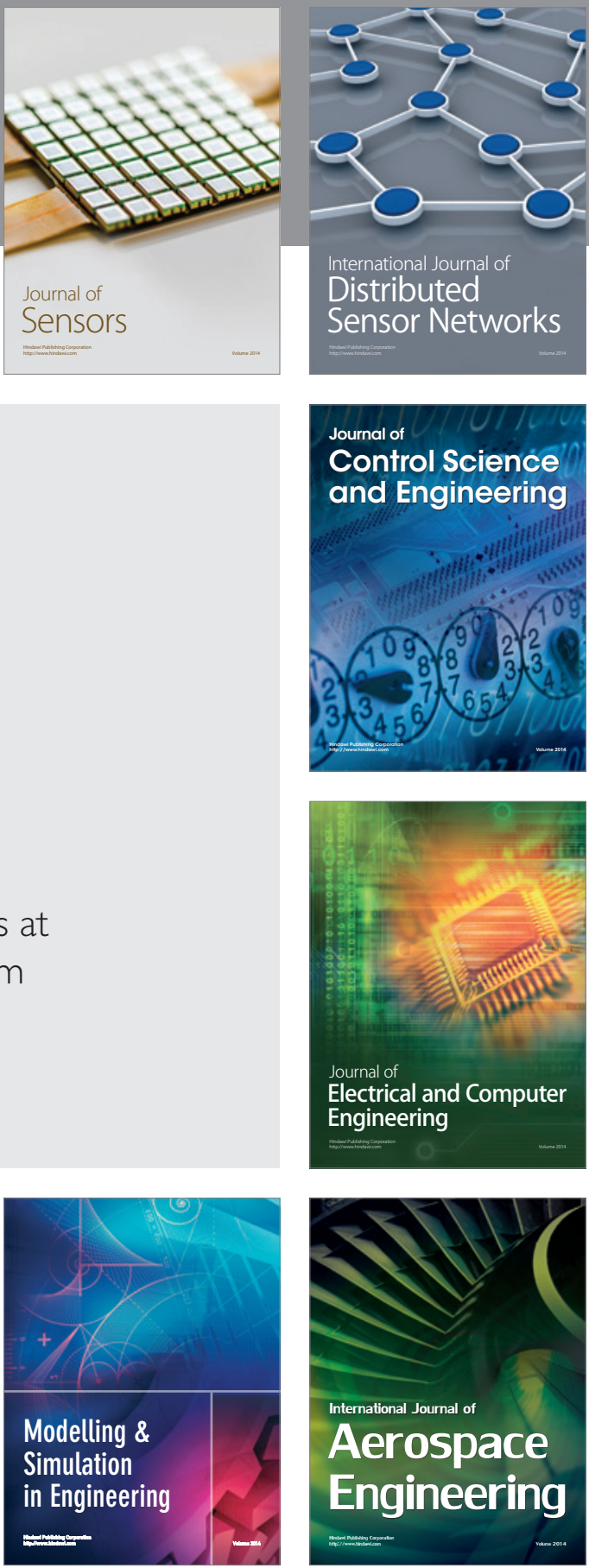

Journal of

Control Science

and Engineering
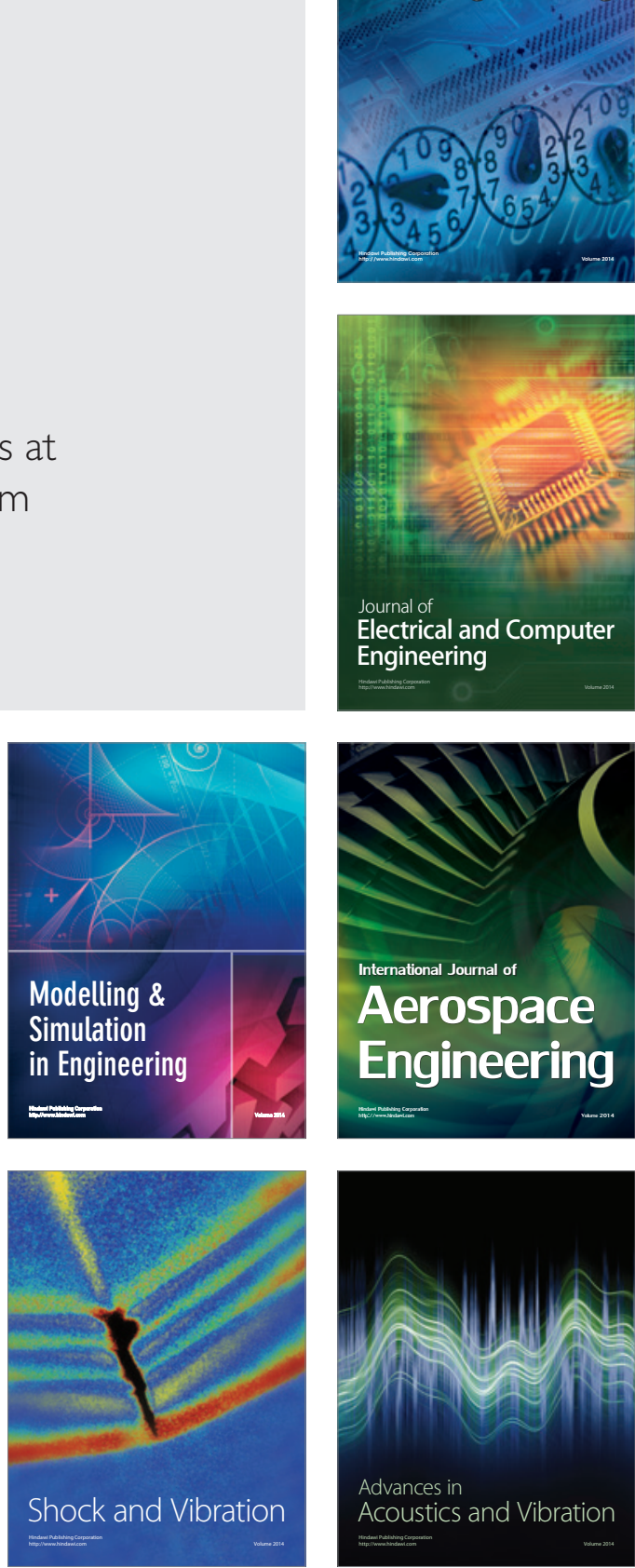